\title{
Restoration of the ATG5-dependent autophagy sensitizes DU145 prostate cancer cells to chemotherapeutic drugs
}

\author{
KE PENG, AIQIN SUN, JUN ZHU, JINYI GAO, YANLIN LI, \\ GENBAO SHAO, WANNIAN YANG and QIONG LIN \\ Department of Basic Medicine, School of Medicine, Jiangsu University, \\ Zhenjiang, Jiangsu 212013, P.R. China
}

Received November 3, 2020; Accepted May 17, 2021

DOI: 10.3892/ol.2021.12899

\begin{abstract}
Autophagy serves an important role in cancer cell survival and drug resistance. In the present study, the prostate cancer DU145 cell line was used, which lacks autophagy related 5 (ATG5) expression and is defective in induction of ATG5-dependent autophagy. The aim of the study was to examine the effects of the restoration of autophagy on cell proliferation and migration, and to assess the cytotoxicity caused by chemotherapeutic drugs, using microscopic, wound-healing, western blot and apoptotic assays. The restoration of the autophagic activity in DU145 cells by the overexpression of ATG5 enhanced the cell proliferation and migration rates. Notably, restoration of the ATG5-dependent autophagy in DU145 cells significantly increased the cytotoxic effects of the chemotherapeutic drugs, docetaxel and valproic acid, and the endoplasmic reticulum stress inducers, brefeldin A, tunicamycin and thapsigargin. The present study provides a novel perspective on the role of ATG5-dependent autophagy in drug resistance and chemotherapy.
\end{abstract}

\section{Introduction}

Autophagy is an important cellular degradation process that is used as a cell compensatory mechanism to various stress conditions, such as nutritional starvation, oxidation, DNA replication and endoplasmic reticulum (ER) and bacterial invasion stress (1-7). Autophagy is generally considered as an important mechanism of cell survival. However, excessive autophagic activity causes severe damage of subcellular structures and results in cell death. Therefore, autophagy serves roles in both cell survival and cell death. Currently, the

Correspondence to: Dr Qiong Lin, Department of Basic Medicine, School of Medicine, Jiangsu University, 301 Xuefu Road, Zhenjiang, Jiangsu 212013, P.R. China

E-mail: qlin@ujs.edu.cn

Key words: prostate cancer, autophagy, autophagy related 5, cytotoxicity, docetaxel, endoplasmic reticulum stressors mechanisms underlying the autophagic regulation in cell death and survival are not fully understood.

The role of autophagy in antitumor drug resistance has been extensively studied (8-10). It is well documented that autophagy is an important process for cancer cell drug resistance, as determined by studies using both targeted therapy and chemotherapy-based approaches $(11,12)$. Therefore, autophagy is considered as a cellular process that promotes tumor cell survival and resistance to antitumor drugs. Currently, suppression of autophagy has emerged as a novel strategy in cancer therapy, which has been applied in multiple types of cancer, such as renal, liver and prostate cancer (13-16).

In the present study, the prostate cancer DU145 cell line was used, which lacks the expression of autophagy related 5 (ATG5) and is therefore defective in ATG5-dependent autophagy $(17,18)$. The experiments aimed to determine the effects of ATG5-dependent autophagy on cell proliferation and migration, and the cytotoxicity of chemotherapeutic drugs by the overexpression of ATG5 in DU145 cells. The results indicated that restoration of autophagy by overexpression of ATG5 enhanced the cytotoxic effects of the chemotherapeutic drugs, docetaxel and valproic acid (VPA), and of the ER stress inducers, brefeldin A, tunicamycin and thapsigargin. The data demonstrated the role of the ATG5-dependent autophagy in the chemotherapeutic drug resistance of cancer cells.

\section{Materials and methods}

Materials. Anti-ATG5 (C-1; cat. no. sc-133158; 1:500), anti-MAP-LC3- $\beta$ (G-9; cat. no. sc-376404; 1:500) and anti-sequesteome 1 (SQSTM1; D-3; cat. no. SC-28359; 1:500) primary antibodies were purchased from Santa Cruz Biotechnology, Inc. Anti- $\beta$-actin (ACTB; cat. no. 100166-MM10; 1:500) primary antibody was obtained from Sino Biological, Inc., and the anti-clathrin primary antibody was obtained from Covance, Inc. The PCR primers were synthesized by Sangon Biotech Co., Ltd. Atorvastatin calcium was purchased from Sigma-Aldrich (Merck KGaA). Rapamycin and bortezomib were purchased from LC Laboratories. Chloroquine was obtained from Cell Signaling Technology, Inc. EGF was obtained from PeproTech, Inc., and docetaxel from Absin Bioscience Inc. Valproic acid (VPA), brefeldin A, tunicamycin and thapsigargin were obtained 
from APeXBIO Technology LLC. The Annexin V-FITC apoptosis detection kit (cat. no. AD10) was purchased from Dojindo Molecular Technologies, Inc. All cancer cell lines were purchased from the American Type Culture Collection.

Cell culture and treatment. The prostate cancer cell lines, DU145, PC3 and 22RV1, and the lung cancer cell lines, A549, NCI-H1650 and NCI-H1975, were cultured in DMEM (Wisent, Inc.; cat. no. 319-005-CL) supplemented with 10\% heat-inactivated FBS (Shanghai ExCell Biology, Inc.) and $100 \mathrm{U} / \mathrm{ml}$ penicillin and streptomycin. The cells were incubated at $37^{\circ} \mathrm{C}$ with $5 \% \mathrm{CO}_{2}$. Treatment with the chemicals, inhibitors or EGF was performed at the concentrations and time points prior to the cell harvest as indicated in the figure legends.

Cell lysate preparation and immunoblotting. The preparation of the cell lysates was performed on ice. The cells were rinsed once with cold PBS following removal of the culture medium, lysed using the ice-cold mammalian cell lysis buffer (100 mM NaCl, 40 mM Hepes, pH 7.4, 25 mM glycerol phosphate, $1 \%$ Triton X-100, $1 \mathrm{mM}$ EDTA, $1 \mathrm{mM}$ sodium orthovanadate, $10 \mu \mathrm{g} / \mathrm{ml}$ leupeptin and $10 \mu \mathrm{g} / \mathrm{ml}$ aprotinin) and incubated on rocking plates at $4^{\circ} \mathrm{C}$ for $30 \mathrm{~min}$. The cell lysates were centrifuged at $15,000 \mathrm{x}$ g in a microcentrifuge for $15 \mathrm{~min}$ at $4^{\circ} \mathrm{C}$ before use. The SDS-PAGE lysate samples were prepared by addition of $5 \mathrm{X}$ SDS sample buffer directly to the lysates, followed by vortexing and denaturation at $100^{\circ} \mathrm{C}$ for 5-10 min. Following electrophoresis on SDS gels (10-14\%), the separated proteins were transferred to a PVDF membrane (EMD Millipore; cat. no. IPFL00010). The membrane was blocked with 1\% BSA (Sigma-Aldrich; Merck KGaA) for $1 \mathrm{~h}$ at $22^{\circ} \mathrm{C}$ and incubated with the aforementioned primary antibodies (anti-ATG5, anti-SQSTM1, anti-LC3 and anti-ACTB) overnight at $4^{\circ} \mathrm{C}$. Following washing of the membrane 4 times with $1 \mathrm{X}$ TBS-Tween-20 (containing $0.1 \%$ Tween-20) for $7 \mathrm{~min}$, the membrane was incubated with HRP-conjugated secondary antibodies $(1: 10,000$; Thermo Fisher Scientific, Inc.; anti-mouse cat. no. 31430; anti-rabbit. cat. no. 31460) for $2 \mathrm{~h}$ at $22^{\circ} \mathrm{C}$. The protein bands were visualized using the Western lightning ECL Detection kit (Beyotime Institute of Biotechnology).

Immunofluorescence staining. The cells were cultured in a 12-well plate on coverslips (MatTek) to 50-70\% confluence. The cells were rinsed with cold PBS twice, fixed with 4\% paraformaldehyde at $22^{\circ} \mathrm{C}$ for $30 \mathrm{~min}$ and permeabilized with $0.5 \%$ Triton X-100 in PBS at $22^{\circ} \mathrm{C}$ for $20 \mathrm{~min}$. Following washing with PBS three times, the cells were incubated with the aforementioned primary antibodies (anti-LC3 or anti-ATG5; 1:100) at $4^{\circ} \mathrm{C}$ overnight. The cells were washed with PBS three times and incubated with a Texas red-conjugated (for anti-ATG5) or an Oregon green-conjugated (for anti-LC3) secondary antibody (1:500; Thermo Fisher Scientific, Inc.; cat. nos. T6390 and O6381, respectively) and DAPI $(1: 10,000)$ at $37^{\circ} \mathrm{C}$ for 1-2 h. Following washing with PBS three times, the coverslips containing cells with fluorescent labels were mounted on microscopic slides and their images were captured using a Zeiss LSM710 confocal microscope (Zeiss AG; cat. no. MA 01960; magnification, $\mathrm{x} 600$ ).
Construction of the ATG5 plasmid in the lentiviral expression vector. The human ATG5 cDNA was subcloned into the FUW-HA lentiviral expression vector (Addgene, Inc.) to establish stable ATG5-overexpressing DU145 cell lines. The ATG cDNA was amplified from a human cDNA library with primers for hATG5-forward-BamH1 (5'-AATCGACTGGATCCATGA CAGATGACAAAGATGT-3') and for hATG5-reverse-EcoR1 (5'-ATCGACGAATTCTCAATCTGTTGGCTGTGG-3') by PCR and inserted into the BamHI/EcoR1 sites of the FUW-HA lentiviral expression vector. PCR was performed using MegaFi ${ }^{\mathrm{TM}}$ Fidelity 2X PCR MasterMix (Applied Biological Materials, Inc.; cat. no. G897) using the following conditions: 5 min at $94^{\circ} \mathrm{C}$ for initial denaturation followed by 22 cycles of $30 \mathrm{sec}$ at $94^{\circ} \mathrm{C}$ for denaturation, $30 \mathrm{sec}$ at $56^{\circ} \mathrm{C}$ for annealing and $30 \mathrm{sec}$ at $72^{\circ} \mathrm{C}$ for elongation, and $10 \mathrm{~min}$ at $72^{\circ} \mathrm{C}$ for final extension.

Lentiviral particle package and infection. The lentiviral plasmid (FUW-HA-ATG5; $1.5 \mu \mathrm{g}$ ) was co-transfected with the psPAX2 (Addgene, Inc.; cat. no. 12260; $1.0 \mu \mathrm{g}$ ) and the pMD2.G (Addgene, Inc.; cat. no. 12259; $0.5 \mu \mathrm{g}$ ) packaging plasmids into 293T cells using Lipofectamine ${ }^{\circledR} 2000$ Transfection reagent (Thermo Fisher Scientific, Inc.; cat no. 11668030) for 8-12 h. The culture medium containing viral particles was collected every $24 \mathrm{~h}$ thrice. The medium was centrifuged at $200 \mathrm{x}$ g for $5 \mathrm{~min}$ at $22^{\circ} \mathrm{C}$ and used for infecting DU145 cells in the presence of $6 \mu \mathrm{g} / \mathrm{ml}$ polybrene (Sigma-Aldrich; Merck KGaA). The infected DU145 cells were selected by $2.5 \mu \mathrm{g} / \mathrm{ml}$ puromycin for three days to obtain the ATG5-overexpressing cell line.

Cell proliferation and migration assays. A total of $2.5 \times 10^{4}$ DU145 cells [transfected with the empty vector control FUW-Mr2tTA (Addgene, Inc.; cat. no. 20342) or FUW-HA-ATG5] were seeded in each well of a 12-well culture plate. Following culture for $24,48,72$ or $96 \mathrm{~h}$, the cells were counted using a light phase microscope (magnification, $x 200$ ) with a hemocytometer. The cell proliferation was evaluated by the increase in cell number. The proliferation assay was repeated at least three times. Cell migration was determined using a wound healing assay as described previously (19). Briefly, DU145 cells $\left(1 \times 10^{6}\right)$ were seeded into 6-well plates and cultured in the standard culture medium (DMEM plus $10 \%$ FBS). When the cells reached 90-95\% confluence, a straight scratch line was made in the cell monolayer using a pipette tip. The cells were subsequently cultured for $36 \mathrm{~h}$ at $37^{\circ} \mathrm{C}$ in the standard culture medium with $10 \%$ FBS for sustaining normal cell migration during wound healing (19). For stimulation of cell migration using EGF, EGF $(100 \mathrm{ng} / \mathrm{ml})$ was directly added into the culture medium during the migration assay. Images of the migrated cells in the scratched zone were captured using a light phase microscope (magnification, $x 100)$. The recovered area of the migrated cells in the scratched zone was quantified using ImageJ software (Version 1.53; National Institutes of Health) and was used for evaluation of the migration rate.

Apoptosis assay. DU145 cells $\left(5 \times 10^{4}\right)$ were seeded in each well of a 12-well culture plate. Following incubation of the cells for $16 \mathrm{~h}$, they were treated with docetaxel $(30 \mathrm{nM})$ and thapsigargin $(250 \mathrm{nM})$ for $48 \mathrm{~h}$ at $37^{\circ} \mathrm{C}$. Following removal of the culture medium, the cells were rinsed with PBS and 
A

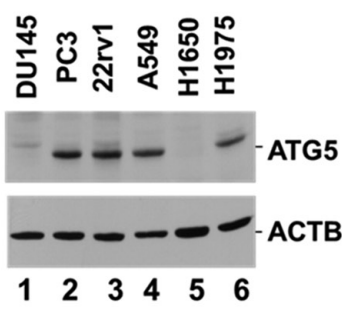

B

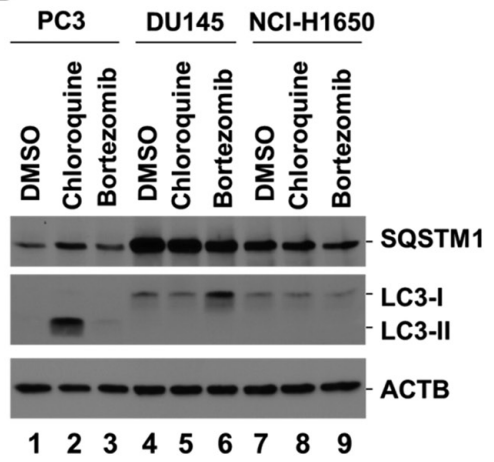

C

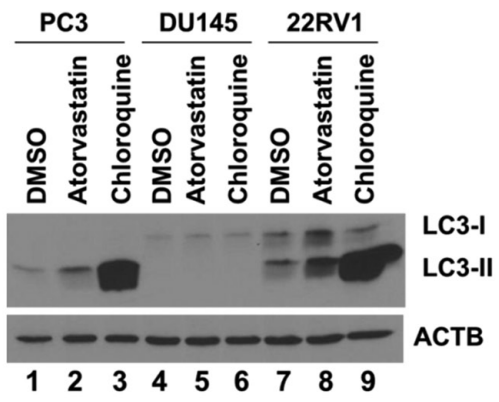

Figure 1. DU145 cells lack ATG5 expression and are defective in the induction of autophagy. (A) Detection of ATG5 protein expression in cell lysates derived from three prostate cancer cell lines, DU145, PC3 and 22RV1, and three lung cancer cell lines, A549, NCI-H1650 and NCI-H1975, using western blotting. (B) Detection of LC3-II and SQSTM1 protein expression in PC3, DU145 and NCI-H1650 cell lysates following treatment of the corresponding cells with the lysosomal inhibitor chloroquine $(50 \mu \mathrm{M})$ or the proteasomal inhibitor bortezomib $(10 \mu \mathrm{M})$ for $24 \mathrm{~h}$. (C) Detection of LC3-II protein expression in PC3, DU145 and 22RV1 cell lysates following treatment of the cells with the lysosomal inhibitor chloroquine (50 $\mu \mathrm{M})$ or the hydroxylmethylglutaryl Co-enzyme A reductase inhibitor atorvastatin $(10 \mu \mathrm{M})$ for $48 \mathrm{~h}$. $\beta$-actin was used as a loading control. ATG5, autophagy related 5; SQSTM1, sequesteome 1.

incubated with $100 \mu 11 \mathrm{X}$ Annexin $\mathrm{V}$ binding solution and $5 \mu \mathrm{l}$ Annexin V-FITC per well at $22^{\circ} \mathrm{C}$ for $15 \mathrm{~min}$. The stained apoptotic cells were observed and quantified using a Nikon Eclipse TE2000 inverted fluorescence microscope (Nikon Corporation; cat. no. NY 1174; magnification, x100).

Statistical analysis. The data from three independent experiments were analyzed with the statistical software SPSS (version 19.0; IBM Corp.) using the Student's t-test (unpaired). The data were presented as the mean \pm standard deviation. $\mathrm{P}<0.05$ was considered to indicate a statistically significant difference.

\section{Results}

Prostate cancer DU146 cell line is defective in the induction of autophagy due to loss of ATG5 expression. To identify specific autophagy-defective cancer cell lines, several prostate and lung cancer cell lines were screened by assessing the expression levels of ATG5. The data indicated that ATG5 was not detectable in DU145 and NCI-H1650 cells (Fig. 1A). DU145 and NCI-H1650 cells have been previously reported as autophagy-defective cell lines, lacking the expression of ATG5 and ATG7 $(17,20)$. To verify whether DU145 and NCI-H1650 were autophagy-defective cell lines, the PC3 and DU145 prostate cancer cells, along with NCI-H1650 lung cancer cells, were incubated with the lysosomal inhibitor chloroquine, which is known to block the autophagic flux and cause accumulation of LC3-II (21). The specific proteasomal inhibitor bortezomib was used as a control. A marked accumulation of LC3-II was observed in PC3 cells following treatment with chloroquine, while no LC3-II expression was detected in either DU145 or NCI-H1650 cells following treatment with chloroquine (Fig. 1B), indicating the lack of autophagic flux for both DU145 and NCI-H1650 cells. In addition, the protein levels of the autophagy receptor SQSTM1, which is degraded by autophagy (1), were markedly higher in DU145 and NCI-H1650 cells than in PC3 cells (Fig. 1B), suggesting a defect in autophagy in both DU145 and NCI-H1650 cells. As the defect in autophagy of the lung cancer NCI-H1650 cells has been well characterized (20), the present study focused on the prostate cancer DU145 cells. The defect in autophagy was further confirmed following treatment of DU145 cells with atorvastatin, a hydroxylmethylglutaryl co-enzyme A reductase inhibitor that has been shown to induce autophagy (22). Treatment of the two ATG5-expressing prostate cancer PC3 and 22RV1 cell lines with atorvastatin induced an increase of LC3-II expression compared with DMSO, but this effect was not noted in DU145 cells (Fig. 1C). Treatment of the cells with chloroquine caused a marked accumulation of LC3-II in PC3 and 22RV1 cells, whereas this effect was not observed in DU145 cells (Fig. 1C). These data confirmed that DU145 cells were defective in the induction of autophagy.

Overexpression of ATG5 restores autophagy in DU145 cells. To confirm that loss of ATG5 expression was the cause for the defect in autophagy of DU145 cells, a lentiviral mammalian expression system was employed and ATG5 expression was restored in the cells (Fig. 2A). Restoration of ATG5 expression induced the expression of the autophagosomal protein LC3-II, indicating that autophagy was resumed (Fig. 2A). In addition, the expression levels of the selective autophagic adaptor protein, SQSTM1, were markedly decreased following ATG5 overexpression, which indicated that the autophagic degradation was active (Fig. 2A). As expected, when the ATG5-expressing cells were treated with chloroquine, LC3-II expression was markedly increased (Fig. 2B), indicating that the autophagic flux was active in the cells. Furthermore, autophagosomes were observed in the ATG5-expressing cells following their treatment with the autophagic inducer rapamycin, whereas these changes were not noted in the vector control cells (Fig. 2C). Overall, these data demonstrated that loss of ATG5 expression was the cause for the defect in autophagy in DU145 cells.

Restoration of autophagy in DU145 cells enhances cell proliferation and migration. The effects of the restoration of autophagy were subsequently examined with regard to DU145 cell proliferation and migration. Ectopic expression of ATG5 significantly increased the cell proliferation rate after 2 days of culture compared with the vector control cells (Fig. 3A). The wound healing assay was used to detect cell migration 

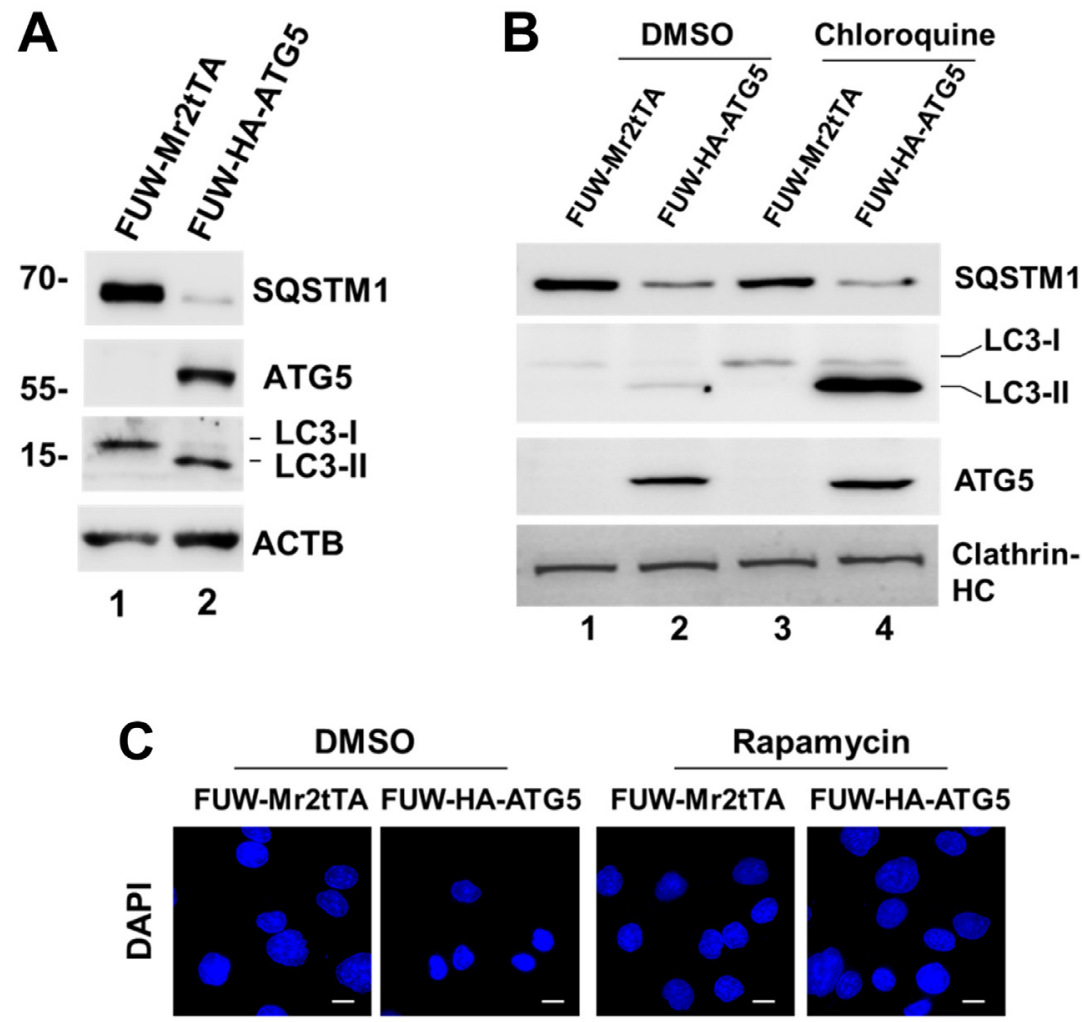

DMSO

\section{Rapamycin}
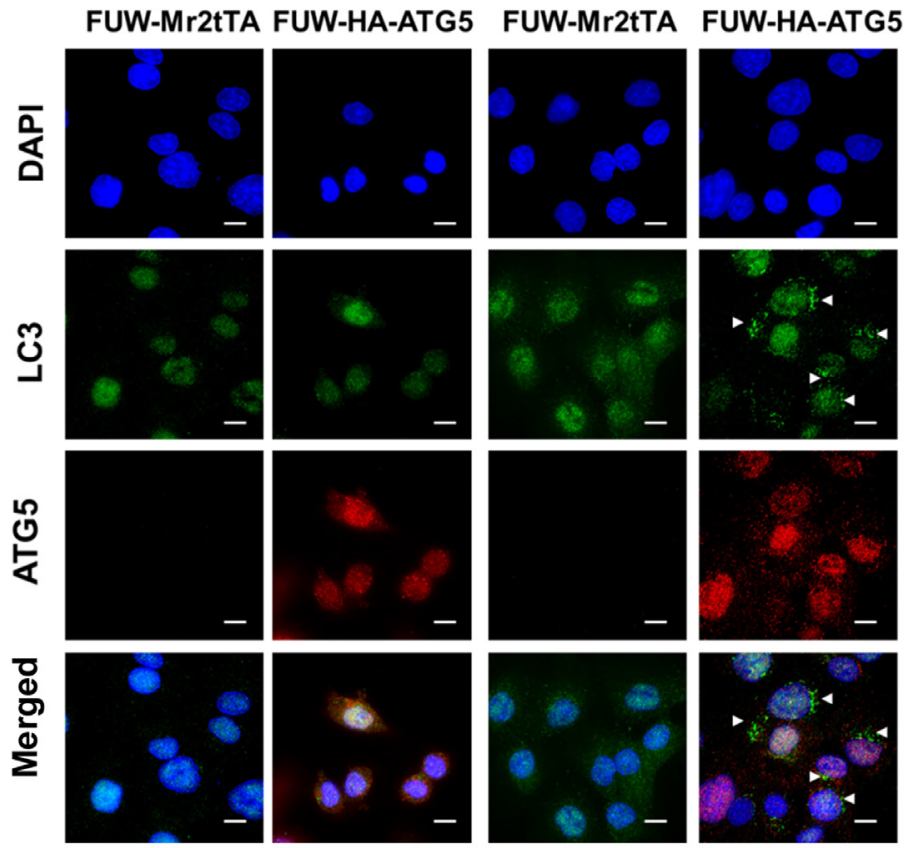

Figure 2. Restoration of ATG5 expression restores ATG5-dependent autophagy in DU145 cells. (A) Ectopic expression of ATG5 in DU145 cells induced formation of the autophagosomal marker protein LC3-II. ATG5 was ectopically expressed in DU145 cells using a lentiviral expression system. The ATG5-expressing and vector control cells were lysed and the expression levels of LC3-II, SQSTM1 and ATG5 were detected by western blot analysis. $\beta$-actin was used as the lysate loading control. (B) Ectopic expression of ATG5 restored the autophagic flux in DU145 cells. The ATG5-expressing cells and the vector control cells were treated with the lysosomal inhibitor chloroquine $(50 \mu \mathrm{M})$ for $12 \mathrm{~h}$ and the expression levels of LC3-II, SQSTM1 and ATG5 were detected by western blot analysis. Clathrin-HC was used as a lysate loading control. (C) Autophagy was induced by the mTOR inhibitor rapamycin in the ATG5-expressing DU145 cells, but not in the vector control cells. The ATG5-expressing and the vector control DU145 cells were treated with rapamycin (1 $\mu \mathrm{M})$ for $12 \mathrm{~h}$. The cells were fixed, and the autophagosomes and ATG5 were visualized by immunofluorescent staining with an anti-LC3 antibody and an anti-ATG5 antibody, respectively. White arrows indicate the induced autophagosomes. Scale bar, $20 \mu \mathrm{m}$. ATG5, autophagy related 5; SQSTM1, sequesteome 1; Clathrin-HC, clathrin heavy chain.

rate in both the control and the ATG5-expressing cell lines. The data indicated that ATG5 overexpression in DU145 cells significantly promoted both the basal and the EGF-stimulated cell migration rate (Fig. 3B and C). These data indicated that restoration of autophagy in DU145 cells facilitated cell proliferation and migration.

Restoration of autophagy enhances the sensitization of DU145 cells to the cytotoxicity caused by the chemotherapeutic drug docetaxel and ER stress-associated drugs. To examine the effects of autophagy on the efficacy of chemotherapeutic drugs and cellular stress resistance, the proliferation rate of the ATG5-expressing cells was compared with that of the vector control cells following treatment with the chemotherapeutic drugs, docetaxel and VPA, or the ER stressors, thapsigargin, tunicamycin and brefeldin A. Notably, $\geq 20 \mathrm{nM}$ docetaxel, $\geq 3.3 \mathrm{mM}$ VPA, $250 \mathrm{nM}$ thapsigargin, $2 \mu \mathrm{M}$ tunicamycin and $500 \mathrm{nM}$ brefeldin A significantly inhibited the proliferation of the ATG5-expressing cells compared with that of the vector control cells (Fig. 4A-C), indicating that restoring autophagy in DU145 increased the sensitivity of the cells to the cellular stress caused by chemotherapeutic drugs or ER stressors. The 


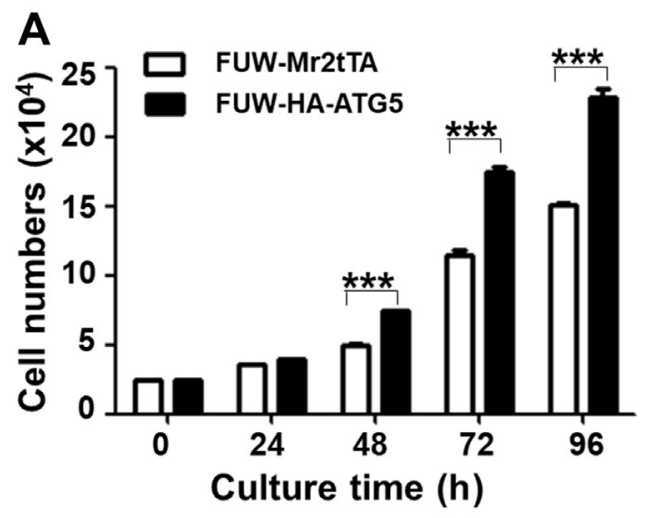

B

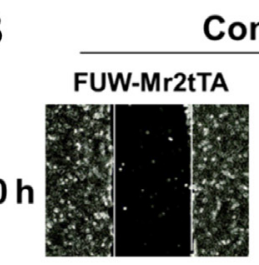

Control
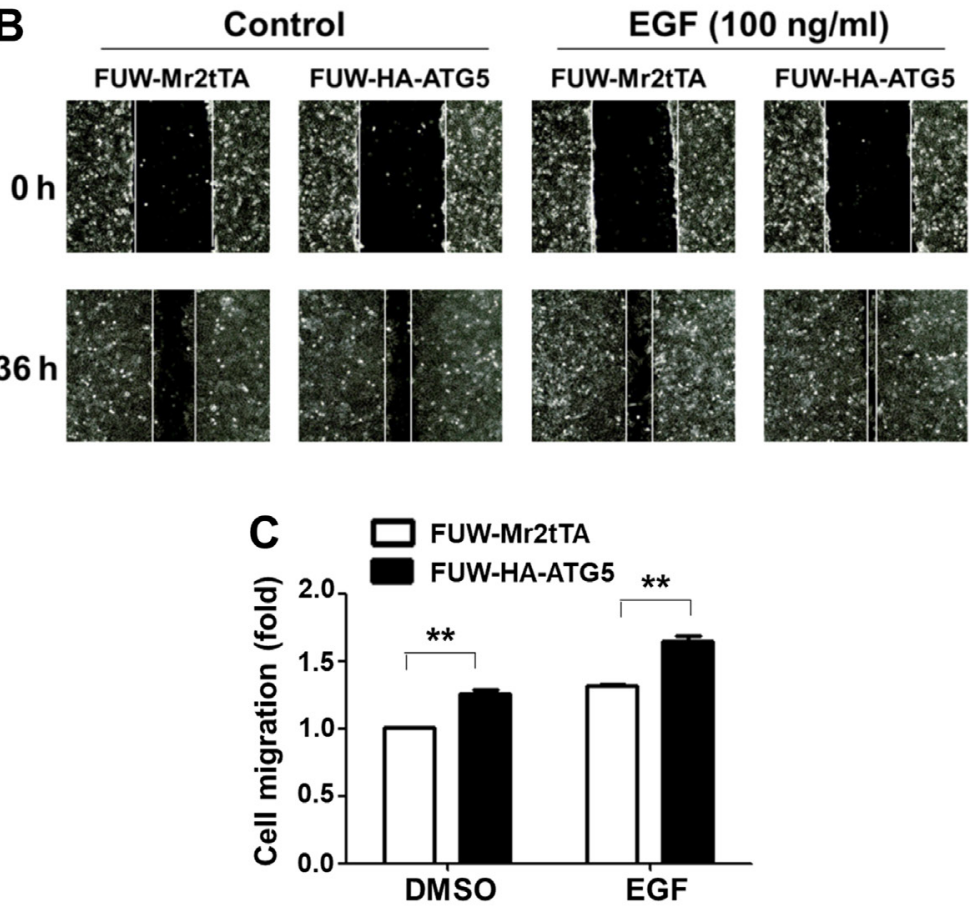

Figure 3. Restoration of the ATG5-dependent autophagy in DU145 cells promotes cell proliferation and migration. (A) ATG5-expressing DU145 cells exhibited a higher proliferation rate compared with the vector control cells. The data used for semi-quantification were from three independent experiments. ${ }^{* * *} \mathrm{P}<0.001$. (B and C) ATG5-expressing DU145 cells exhibited a higher migration rate compared with the vector control cells. (B) Wound healing assay images (magnification, $\mathrm{x} 100$ ). (C) Semi-quantification of cell migration was based on three independent experiments using the wound healing assay. ${ }^{* *} \mathrm{P}<0.01$. ATG5, autophagy related 5.

effects of the restored autophagy on cell survival were further investigated following treatment with the chemotherapeutic drug docetaxel and the ER stressor thapsigargin. The treatment of the ATG5-expressing cells with these compounds caused a significant increase in apoptosis compared with that in the vector control cells (Fig. 5A and B), indicating that restoring autophagy in DU145 cells resulted in sensitization to the stress signal-induced apoptosis.

\section{Discussion}

Previous studies have demonstrated that DU145 cells lack expression of ATG5 and are defective in the induction of autophagy $(17,18)$. Restoration of ATG5 reverses the autophagic response to VPA (17). The results of the present study confirmed that DU145 cells lacked ATG5 expression and were defective in ATG5-dependent autophagy. Restoration of ATG5 expression reversed autophagy and enhanced cell proliferation and migration, suggesting that the ATG5-dependent autophagy may benefit the cancer cells and promote cancer progression. Notably, restoration of the ATG5-dependent autophagy in DU145 cells increased the sensitivity of the cells to the cytotoxicity induced by the chemotherapeutic drugs, docetaxel and VPA, and the ER stressors, brefeldin A, tunicamycin and thapsigargin, compared with the effects in the vector control cells, suggesting that ATG5-dependent autophagy sensitized cells to the antitumor effects of the chemotherapeutic drugs and ER stressors. It is well documented that autophagy is an important cellular process required to counteract various stress conditions and maintain cell survival in cancer cells (1-7). It has been proposed to use autophagy inhibitors combined with chemotherapeutic drugs to overcome the drug resistance in cancer (23). However, the present study revealed a novel role of autophagy in sensitization of cancer cells to the cytotoxicity caused by chemotherapeutic drugs or ER stressors, raising a concern when applying the therapeutic strategy of inhibiting autophagy to overcome the chemoresistance in patients with cancer. The current observation of the sensitization to 

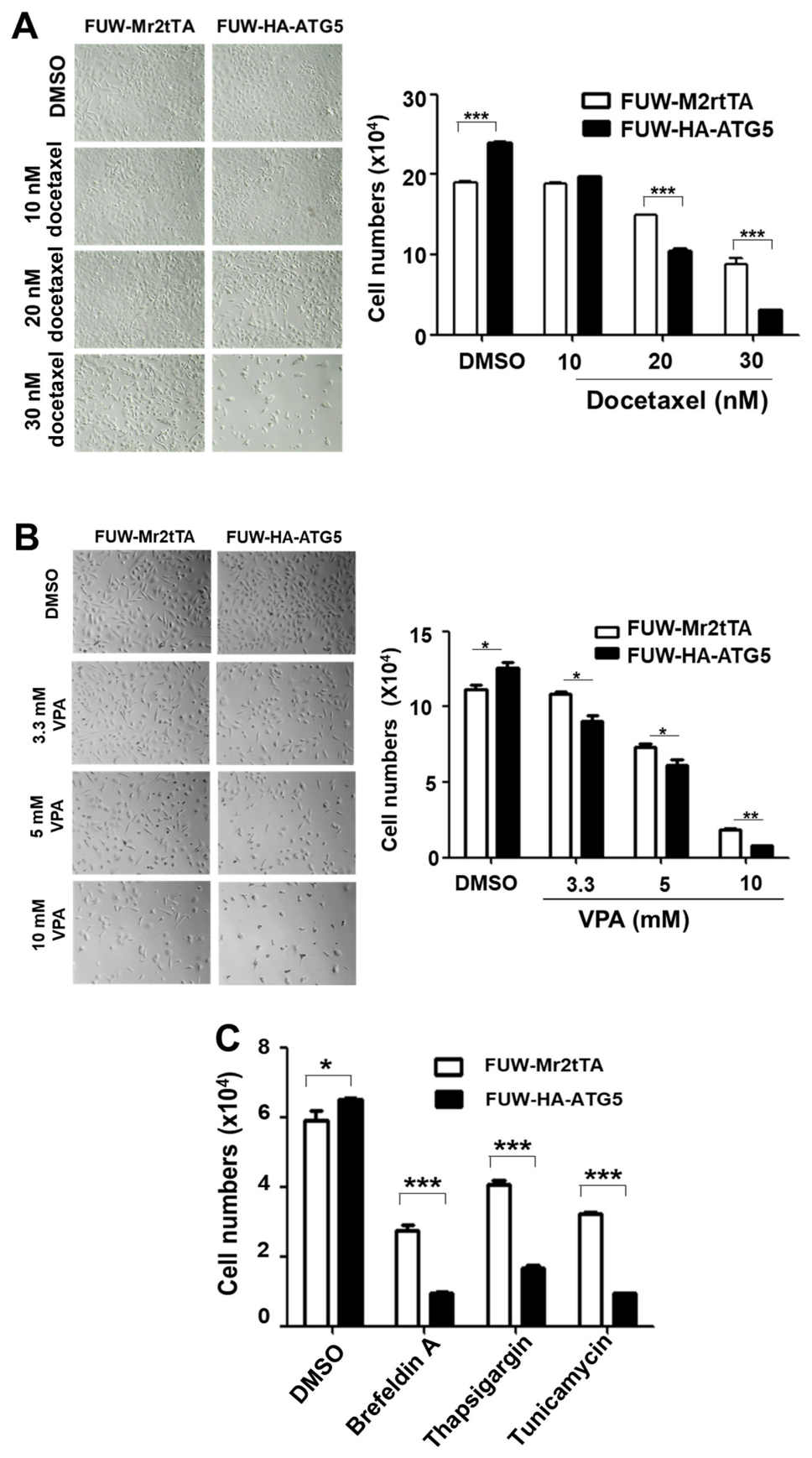

Figure 4. Restoration of ATG5-dependent autophagy in DU145 cells sensitizes them to the cytotoxicity of chemotherapeutic drugs and to ER stressors. (A) Light phase microscopic images of cells treated with the indicated concentrations of docetaxel for $72 \mathrm{~h}$ (left panel; magnification, x200) and semi-quantification of the inhibition of proliferation by docetaxel treatment in ATG5-expressing or vector control cells (right panel). (B) Light phase microscopic images of the cells treated with the indicated concentrations of VPA for $48 \mathrm{~h}$ (left panel; magnification, $\mathrm{x} 200$ ) and semi-quantification of the inhibition of proliferation by docetaxel treatment in ATG5-expressing or vector control cells (right panel). (C) Semi-quantification of the inhibition of proliferation by the ER stressors brefeldin A $(500 \mathrm{nM})$, tunicamycin $(2.0 \mu \mathrm{g} / \mathrm{ml})$ and thapsigargin $(250 \mathrm{nM})$ for $24 \mathrm{~h}$ in the ATG5-expressing or the vector control cells. In all the quantification figures, the data were from three independent experiments. ${ }^{*} \mathrm{P}<0.05 ;{ }^{* *} \mathrm{P}<0.01 ;{ }^{* * *} \mathrm{P}<0.001$. ATG5, autophagy related 5; VPA, valproic acid

cytotoxicity of chemotherapeutic drugs or ER stressors by autophagy was limited to DU145 cells. To conclude the role of autophagy in sensitizing cytotoxicity of chemotherapeutic drugs or ER stressors in prostate cancer, more prostate cancer cell lines should be investigated.

Notably, the current finding on the sensitization effect of the restored autophagy on the cytotoxicity of VPA has not been observed in a previous study (17). This discrepancy may result from the difference in re-expression level of ATG5 in DU145 cells between the studies. It seems that the restored level of ATG5 in DU145 cells in the present study was higher than that in a study by Ouyang et al (17), which may yield differential sensitivity of the restored autophagy response to cytotoxicity of VPA. Further studies may need to establish the connection between intensity of autophagic activity and sensitivity of cancer cells to chemotherapeutic drugs.

The current study did not explain the mechanism by which DU145 and NCI-H1650 cells, which are defective in the ATG5-dependent autophagy, were able to maintain cellular homeostasis and respond to various stress signals. These cells 


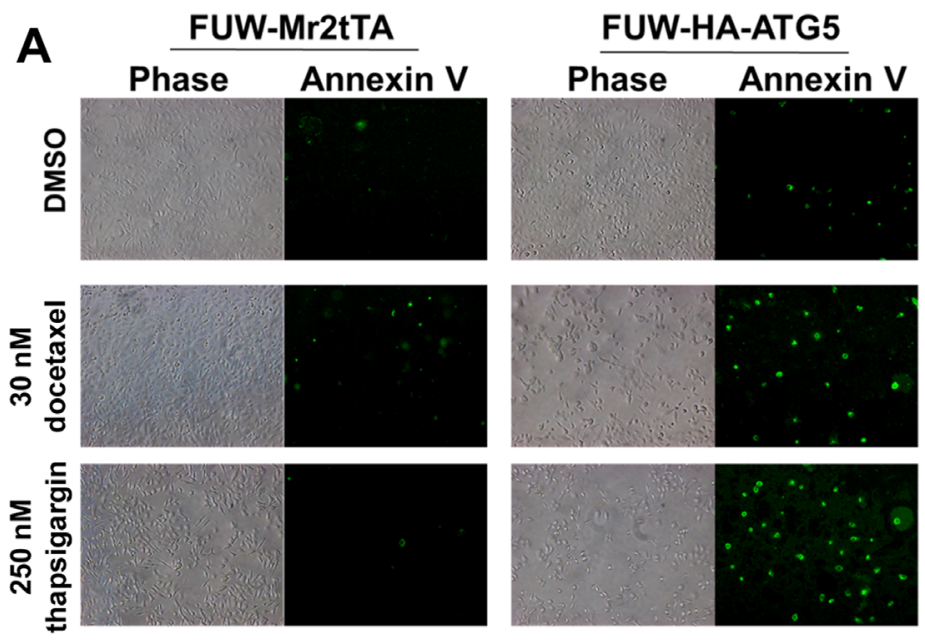

B

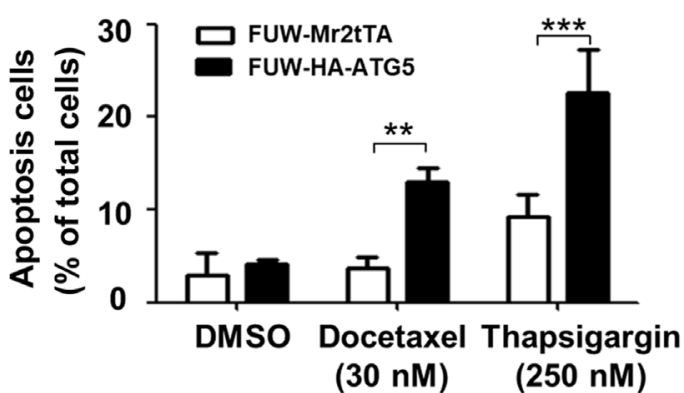

Figure 5. Restoration of ATG5-dependent autophagy in DU145 cells enhances apoptosis induced by docetaxel or thapsigargin. (A) ATG5-expressing or vector control cells were treated with the indicated concentration of docetaxel or thapsigargin for $48 \mathrm{~h}$. The number of apoptotic cells was visualized using fluorescent staining with FITC-conjugated Annexin V. 'Phase' refers to light microscopic images (magnification, x100). 'Annexin V' refers to images of the FITC-Annexin V fluorescence (magnification, x100). The FITC-Annexin V fluorescent dots represent the apoptotic cells. (B) Semi-quantification of the apoptotic cells. The data were from three independent experiments. ${ }^{* *} \mathrm{P}<0.01 ;{ }^{* * *} \mathrm{P}<0.001$. ATG5, autophagy related 5 .

may have developed an ATG5-independent autophagic pathway in order to sustain their autophagic function. Previous studies have identified an ATG5-independent autophagic pathway (known as alternative autophagy) that is dependent on RAB9A member RAS oncogene family and utilizes the endosome- or Golgi-derived vesicles for substrate degradation $(24,25)$. A previous study has revealed that this alternative autophagic pathway serves an important role in genotoxic stress (26). Additionally, it has been proposed that alternative autophagy is a complementary autophagic process to the ATG5-dependent autophagy (conventional autophagy) (25). However, whether alternative autophagy is able to replace the full function of ATG5-dependent autophagic activities remains unknown.

The molecular mechanism underlying the effects of the restored ATG5-dependent autophagy on the cytotoxicity caused by the chemotherapeutic drugs and the ER stressors is not known. It may be possible that the restored ATG5-dependent autophagy may interfere with the established ATG5-independent autophagy and may cause the cells to be more sensitive to the stresses induced by the chemotherapeutic drugs and the ER stressors compared with the effects noted in the parent cells. Stresses were more efficient in activating apoptosis in the ATG5-expressing DU145 cells than in the vector control cells. Further clarification of the signaling events mediated by the restored ATG5-dependent autophagy would enhance the understanding of the role of this process in sensitizing cells to the cytotoxicity of the chemotherapeutic drugs and stressors. In conclusion, the current approach may aid the development of precise autophagic-associated therapeutic strategies for prostate cancer therapy.

\section{Acknowledgements}

Not applicable.

\section{Funding}

The present study was funded by the National Natural Science Foundation of China (grant nos. 81372208, 81871888 and 81472558).

\section{Availability of data and materials}

The datasets used and/or analyzed during the current study are available from the corresponding author on reasonable request.

\section{Authors' contributions}

KP performed the majority of the experiments. AS performed the immunofluorescence staining analysis. JZ performed the experiments on screening cancer cell lines for ATG5 expression. JG performed the cell culture experiments. YL 
performed the experiments on ATG5 cDNA cloning. GS participated in the experimental data analysis. WY participated in the experimental design, data analysis and manuscript preparation. QL conceived the study and participated in the experimental design analysis of the data and preparation of the manuscript. KP and QL confirm the authenticity of all the raw data. All authors have read and approved the final manuscript.

\section{Ethics approval and consent to participate}

Not applicable.

\section{Patient consent for publication}

Not applicable.

\section{Competing interests}

The authors declare that they have no competing interests.

\section{References}

1. Klionsky DJ, Abdelmohsen K, Abe A, Abedin MJ, Abeliovich H, Acevedo Arozena A, Adachi H, Adams CM, Adams PD, Adeli K, et al: Guidelines for the use and interpretation of assays for monitoring autophagy (3rd edition). Autophagy 12: 1-222, 2016.

2. Mizushima N: Autophagy: Process and function. Genes Dev 21 : 2861-2873, 2007.

3. Kiffin R, Bandyopadhyay U and Cuervo AM: Oxidative stress and autophagy. Antioxid Redox Signal 8: 152-162, 2006.

4. Vanzo R, Bartkova J, Merchut-Maya JM, Hall A, Bouchal J, Dyrskjøt L, Frankel LB, Gorgoulis V, Maya-Mendoza A, Jäättelä $\mathrm{M}$ and Bartek $\mathrm{J}$ : Autophagy role(s) in response to oncogenes and DNA replication stress. Cell Death Differ 27: 1134-1153, 2020

5. Yorimitsu T, Nair U, Yang Z and Klionsky DJ: Endoplasmic reticulum stress triggers autophagy. J Biol Chem 281: 30299-30304, 2006.

6. Ogata M, Hino S, Saito A, Morikawa K, Kondo S, Kanemoto S, Murakami T, Taniguchi M, Tanii I, Yoshinaga K, et al: Autophagy is activated for cell survival after endoplasmic reticulum stress. Mol Cell Biol 26: 9220-9231, 2006.

7. Mizushima N: The pleiotropic role of autophagy: From protein metabolism to bactericide. Cell Death Differ 12 (Suppl 2): S1535-S1541, 2005.

8. Chen S, Rehman SK, Zhang W, Wen A, Yao L and Zhang J: Autophagy is a therapeutic target in anticancer drug resistance. Biochim Biophys Acta 1806: 220-229, 2010.

9. Livesey KM, Tang D, Zeh HJ and Lotze MT: Autophagy inhibition in combination cancer treatment. Curr Opin Investig Drugs 10: 1269-1279, 2009

10. Li X, Zhou Y, Li Y, Yang L, Ma Y, Peng X, Yang S, Liu J and Li H: Autophagy: A novel mechanism of chemoresistance in cancers. Biomed Pharmacother 119: 109415, 2019.
11. Schoenlein PV, Periyasamy-Thandavan S, Samaddar JS, Jackson WH and Barrett JT: Autophagy facilitates the progression of ERalpha-positive breast cancer cells to anti-estrogen resistance. Autophagy 5: 400-403, 2009.

12. Vazquez-Martin A, Oliveras-Ferraros C and Menendez JA: Autophagy facilitates the development of breast cancer resistance to the anti-HER2 monoclonal antibody trastuzumab. PLoS One 4: e6251, 2009.

13. Yang ZJ, Chee CE, Huang S and Sinicrope FA: The role of autophagy in cancer: Therapeutic implications. Mol Cancer Ther 10: 1533-1541, 2011.

14. Jones TM, Carew JS and Nawrocki ST: Therapeutic targeting of autophagy for renal cell carcinoma therapy. Cancers (Basel) 12: 1185,2020

15. Tran E, Chow A, Goda T, Wong A, Blakely K, Rocha M, Taeb S, Hoang VC, Liu SK and Emmenegger U: Context-dependent role of ATG4B as target for autophagy inhibition in prostate cancer therapy. Biochem Biophys Res Commun 441: 726-731, 2013.

16. Lee YG and Jeon TI: Modulation of the autophagy-lysosomal pathway in hepatocellular carcinoma using small molecules. Molecules 25: 1580, 2020.

17. Ouyang DY, Xu LH, He XH, Zhang YT, Zeng LH, Cai JY and Ren S: Autophagy is differentially induced in prostate cancer LNCaP, DU145 and PC-3 cells via distinct splicing profiles of ATG5. Autophagy 9: 20-32, 2013.

18. Cristofani R, Montagnani Marelli M, Cicardi ME, Fontana F, Marzagalli M, Limonta P, Poletti A and Moretti RM: Dual role of autophagy on docetaxel-sensitivity in prostate cancer cells. Cell Death Dis 9: 889, 2018.

19. Sun A, Yu G, Dou X, Yan X, Yang W and Lin Q: Nedd4-1 is an exceptional prognostic biomarker for gastric cardia adenocarcinoma and functionally associated with metastasis. Mol Cancer 13: 248, 2014.

20. Mandelbaum J, Rollins N, Shah P, Bowman D, Lee JY, Tayber O, Bernard H, LeRoy P, Li P, Koenig E, et al: Identification of a lung cancer cell line deficient in atg7-dependent autophagy. Autophagy: Jun 19, 2015 (Epub Ahead of Print).

21. Iwai-Kanai E, Yuan H, Huang C, Sayen MR, Perry-Garza CN, Kim L and Gottlieb RA: A method to measure cardiac autophagic flux in vivo. Autophagy 4: 322-329, 2008.

22. Parikh A, Childress C, Deitrick K, Lin Q, Rukstalis D and Yang W: Statin-induced autophagy by inhibition of geranylgeranyl biosynthesis in prostate cancer PC3 cells. Prostate 70: 971-981, 2010.

23. Xiao M, Benoit A, Hasmim M, Duhem C, Vogin G, Berchem G, Noman MZ and Janji B: Targeting cytoprotective autophagy to enhance anticancer therapies. Front Oncol 11: 626309, 2021.

24. Nishida Y, Arakawa S, Fujitani K, Yamaguchi H, Mizuta T, Kanaseki T, Komatsu M, Otsu K, Tsujimoto Y and Shimizu S: Discovery of Atg5/Atg7-independent alternative macroautophagy. Nature 461: 654-658, 2009.

25. Shimizu S: Biological roles of alternative autophagy. Mol Cells 41: 50-54, 2018

26. Torii S, Yamaguchi H, Nakanishi A, Arakawa S, Honda S, Moriwaki K, Nakano H and Shimizu S: Identification of a phosphorylation site on Ulk1 required for genotoxic stress-induced alternative autophagy. Nat Commun 11: 1754, 2020.

This work is licensed under a Creative Commons Attribution-NonCommercial-NoDerivatives 4.0 International (CC BY-NC-ND 4.0) License. 